\title{
Repetibilidade da mensuração de imagens das características de carcaça obtidas por ultrassonografia em fêmeas Nelore ${ }^{1}$
}

\section{Maria Eugênia Zerlotti Mercadante ${ }^{2}$, Saulo da Luz e Silva ${ }^{3}$, Mauro Sartori Bueno ${ }^{4}$, Jaime Urdapilleta Tarouco ${ }^{5}$, Marcos Jun Iti Yokoo ${ }^{6}$}

\author{
1 Projeto financiado pela FAPESP. \\ 2 Estação Experimental de Zootecnia de Sertãozinho, APTA, SAA, Sertãozinho, SP. Bolsista do CNPq. \\ ${ }^{3}$ Faculdade de Zootecnia e Engenharia de Alimentos, USP, Pirassununga, SP. \\ ${ }^{4}$ Instituto de Zootecnia, Nova Odessa, APTA, SAA, SP. \\ ${ }^{5}$ Faculdade de Agronomia, Universidade Federal do Rio Grande do Sul, Porto Alegre, RS \\ ${ }^{6}$ Faculdade de Ciências Agrárias e Veterinárias, UNESP, Jaboticabal, SP.
}

RESUMO - Avaliou-se a repetibilidade da mensuração de imagens de ultrassom da área do músculo longissimus dorsi (AOL) e das espessuras de gordura subcutânea do lombo (EGL) e da garupa (EGG). Imagens de ultrassom tomadas no lombo (entre a 12a e a 13a costela) e na garupa (entre os músculos gluteus medium e biceps femoris) de novilhas Nelore de 14 a 22 meses de idade foram classificadas em aceitáveis, marginais e rejeitáveis. As imagens aceitáveis e marginais foram mensuradas duas vezes por três técnicos em diferentes níveis de treinamento. Foram estimadas as repetibilidades entre e dentro de técnicos por classe de qualidade da imagem, para determinação do efeito da qualidade da imagem e do técnico no valor absoluto da diferença entre a primeira e a segunda mensuração dessas características. A repetibilidade para as imagens aceitáveis foi maior que para imagens marginais, tanto entre como dentro de técnicos. Na análise da diferença absoluta entre a primeira e a segunda interpretação, foram significativos os efeitos de técnico para AOL e EGL e de classe de qualidade da imagem para AOL. Em geral, o técnico com maior experiência apresentou maiores valores de repetibilidade. É recomendável que a mensuração de imagens de animais de mesmo grupo contemporâneo seja feita por um único técnico.

Palavras-chave: área do músculo longissimus dorsi, bovinos de corte, espessura de gordura subcutânea, qualidade da imagem, ultrassom

\section{Repeatability of ultrasound image measurements of carcass traits in Nellore cattle}

\begin{abstract}
The repeatability of ultrasound image measurements of the longissimus dorsi muscle (AOL) and of the rumpfat (EGG) and backfat (EGL) subcutaneous thickness was evaluated. Ultrasound images taken from the back (between $12^{\text {th }}$ and $13^{\text {th }}$ ribs) and from the rump (between gluteus medium and biceps femoris muscles) of Nelore heifers at 14 and 22 months of age were classified as acceptable, marginal and rejected. The acceptable and marginal images were measured twice by three technicians at different levels of training. It was estimated repeatabilities among and within technicians by class of image quality in order to determine effect of image quality and of the technician at the absolute difference value between the first and second measurement of those traits. The repeatability for acceptable images was higher than for marginal images, for both among and within technicians. Regarding to the absolute difference analyses between the first and second interpretation, the effects of the technician for AOL and EGL and quality class of image for AOL were significant. In general, the repeatability was higher for the most experienced technician. It is advisable that measurements of images in the same contemporaneous group should be done by one single technician.
\end{abstract}

Key Words: backfat thickness, beef cattle, image quality, longissimus dorsi muscle area, ultrasound

\section{Introdução}

A seleção de bovinos de corte com maior deposição de gordura subcutânea pode agregar maior valor aos animais e proporcionar melhor remuneração ao produtor, uma vez que alguns frigoríficos já pagam a mais $2 \%$ por carcaças de animais Zebu com até 36 meses de idade (4 dentes), espessura de gordura subcutânea de 3-6 mm e $270 \mathrm{~kg}$ de peso (Dias, 2006). No Brasil, o uso da técnica de ultrassom em bovinos de corte para estimar algumas medidas de carcaça no animal vivo iniciou-se na década de 1990 (Tarouco et al., 1993). Entretanto, foi somente a partir de

Recebido em 22/9/2008 e aprovado em 1/4/2009.

Correspondências devem ser enviadas para: mercadante@iz.sp.gov.br 
2000 que essa tecnologia começou a ser inserida nos programas de melhoramento genético de bovinos de corte, principalmente da raça Nelore (Lôbo et al., 2004). Esse fato aumentou a necessidade de grande número de técnicos treinados, tanto na coleta das imagens no campo, como na mensuração das imagens em laboratório.

Os erros na obtenção das medidas da área do músculo longissimus dorsi e da espessura de gordura subcutânea por ultrassonografia podem ser introduzidos tanto durante a aquisição das imagens como na sua mensuração (Hassen et al., 1998). A repetibilidade da mensuração das imagens é sempre maior dentro de técnicos $(0,86$ a 0,97$)$ que entre técnicos $(0,76)$ e é maior para as medidas de espessura de gordura que para a medida da área do longissimus dorsi (Perkins et al., 1992; Hassen et al., 1998).

Os erros de mensuração das imagens de ultrassom podem ser ocasionados pelo posicionamento do cursor na estrutura anatômica visualizada e podem variar conforme o operador. As estruturas ecogênicas (estruturas com diferentes impedâncias acústicas) que dividem os tecidos têm, frequentemente, dimensões variáveis, o que pode causar diferenças entre operadores no momento da interpretação das imagens e, consequentemente, nos resultados obtidos. A imagem de ultrassom do músculo longissimus dorsi apresenta pontos anatômicos de difícil visualização, como em suas bordas laterais e inferiores, que muitas vezes têm má resolução, o que pode levar a mensurações distintas e diferenças dentro de e entre operadores (Wilson et al., 1999).

A qualidade das imagens é fundamental na acurácia dos resultados obtidos. Desta forma, os protocolos de avaliação de características de carcaça por ultrassonografia recomendam a classificação das imagens em grupos de acordo com sua qualidade: aceitáveis, marginais ou descartáveis (Wilson, 2006).

O objetivo neste estudo foi avaliar a repetibilidade da mensuração de imagens de ultrassom da área do músculo longissimus dorsi (AOL) e das espessuras de gordura subcutâneas do lombo (EGL) e da garupa (EGG), de qualidade aceitável ou marginal, obtidas em fêmeas da raça Nelore.

\section{Material e Métodos}

As imagens foram escolhidas aleatoriamente em um banco de imagens e capturadas em duas épocas: julho de 2007 (122 imagens) e fevereiro de 2008 (202 imagens) por dois técnicos, em novilhas com idade de 14 a 22 meses e peso médio de $275 \pm 55 \mathrm{~kg}$, pertencentes aos rebanhos Nelore da Estação Experimental de Zootecnia de Sertãozinho.
As imagens foram colhidas e gravadas com aparelho de ultrassonografia Pie Medical 401347-Aquila (Esaote Europe B.V.), sonda linear de $18 \mathrm{~cm}$, de 3,5 MHz. Para obtenção das imagens da AOL e da EGL, o transdutor foi colocado perpendicularmente à coluna vertebral entre a $12^{\mathrm{a}}$ e $13^{\mathrm{a}}$ costelas, do lado esquerdo do animal, utilizando-se um acoplador acústico (standoff). Para obtenção da imagem da EGG, o transdutor foi colocado na intersecção dos músculos gluteus medius e biceps femoris, localizados entre o ílio e o ísquio, sem utilização do acoplador acústico, aplicando-se óleo vegetal como acoplante.

Um técnico altamente especializado classificou as 324 imagens em aceitáveis (127 imagens, 39\% do total), marginais (173 imagens, $53 \%$ do total) e rejeitáveis (24 imagens, $8 \%$ do total), de acordo com as descrições de Wilson (2006), e não participou de suas mensurações. As imagens foram classificadas como aceitáveis quando todos os pontos anatômicos (Figura 1) eram visualizados e, como marginais, quando um ou mais dos pontos anatômicos descritos eram de difícil visualização (Figura 2).

Posteriormente, as imagens aceitáveis e marginais foram mensuradas duas vezes, por três técnicos (incluindo os dois que capturaram as imagens), um deles altamente treinado (técnico 2). Na mensuração das imagens, utilizou-se o programa Echo Image Viewer 1.0 (Pie Medical Equipament B.V., 1996) com precisão de uma casa decimal.

A variância da mensuração de determinada imagem foi particionada em variâncias atribuídas ao técnico $\left(\sigma_{\text {tec }}^{2}\right.$, tec $=1,2,3)$, à mensuração $\left(\sigma_{\text {mensur }}^{2}\right.$ leitura $\left.=1,2\right)$, à imagem $\left(\sigma_{\text {imagem }}^{2}\right.$,imagem $\left.=1, \ldots ., N\right)$ e ao resíduo $\left(\sigma_{\mathrm{e}}^{2}\right)$, em que $\mathrm{N}$ é o número total de imagens analisadas para cada característica (AOL, EGL ou EGG).

$$
\mathrm{y}_{\mathrm{ijkl}}=\mu+\mathrm{t}_{\mathrm{i}}+\mathrm{u}_{\mathrm{j}}+\mathrm{v}_{\mathrm{k}}+\mathrm{e}_{\mathrm{ijkl}}
$$
em que: $y_{i j k l}=i j k l$-ésima observação da AOL, EGL ou EGG; $\mu=$ média geral; $t_{i}=$ efeito aleatório do i-ésimo técnico; $\mathrm{u}_{\mathrm{j}}=$ efeito aleatório da $\mathrm{j}$-ésima mensuração; $\mathrm{v}_{\mathrm{k}}=$ efeito aleatório da k-ésima imagem; $\mathrm{e}_{\mathrm{ijkl}}=$ erro residual.

A variância entre imagens refere-se à variação associada a diferenças entre mensurações de diferentes imagens, portanto, é a covariância entre mensurações repetidas de mesma imagem. A variância de técnico representa a variação associada a diferenças na mensuração da imagem por dois técnicos. As variâncias foram estimadas por máxima verossimilhança restrita no procedimento MIXED (SAS, Inst., Inc., Cary, NC), para cada característica em cada tipo de imagem (aceitável e marginal) separadamente. A repetibilidade é definida como a correlação entre medidas repetidas de uma mesma unidade experimental. Esse valor foi calculado entre técnicos, dentro de técnicos e para cada 

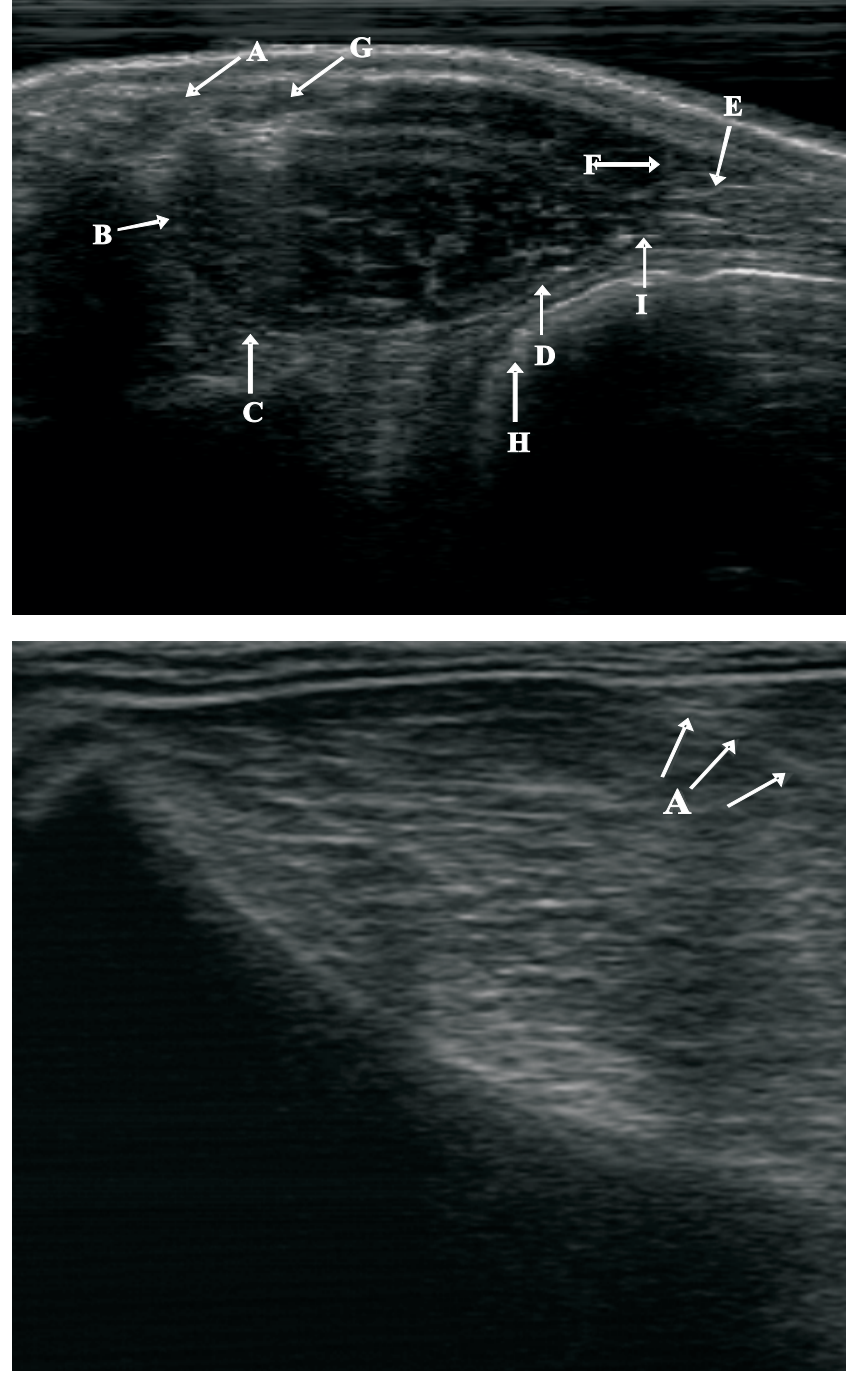

Figura 1 - Pontos anatômicos de uma imagem obtida na região entre a 12 a e 13 a costelas (acima) e classificada como aceitável: A) músculo spinalis dorsi; B) borda medial do músculo longissimus dorsi bem delimitada; C) músculo quadratus lumborum; D) músculos intercostais, os quais estão paralelos; E) músculo longissimus costarum; F) borda lateral do músculo longissimus dorsi bem delimitada; G) "acorn fat" ou gancho de gordura; H) diafragma; I) quebra dos intercostais, que auxilia a identificar a delimitação entre os músculos longissimus dorsi e longissimus costarum; e de uma imagem obtida na região da picanha (abaixo) e classificada como aceitável: A) divisão entre os músculos biceps femoris e gluteus medius bem delimitada.

técnico separadamente, por classe de qualidade da imagem como:

$$
t=\frac{\sigma_{\text {imagem }}^{2}}{\sigma_{\text {imagem }}^{2}+\sigma_{\text {tec }}^{2}+\sigma_{\text {mensur }}^{2}+\sigma_{e}^{2}},
$$

em que: $\mathrm{t}=$ repetibilidade, $\sigma_{\text {imagem }}^{2}=$ variância entre imagens, $\sigma_{\text {tec }}^{2}=$ variância de técnico, $\sigma_{\text {mensur }}^{2}=$ variância da ordem da mensuração; e $\sigma_{\mathrm{e}}^{2}=$ variância residual. A variância de $\mathrm{t}\left(\sigma_{\mathrm{t}}^{2}\right)$ foi calculada segundo Falconer \& Mackay (1996):
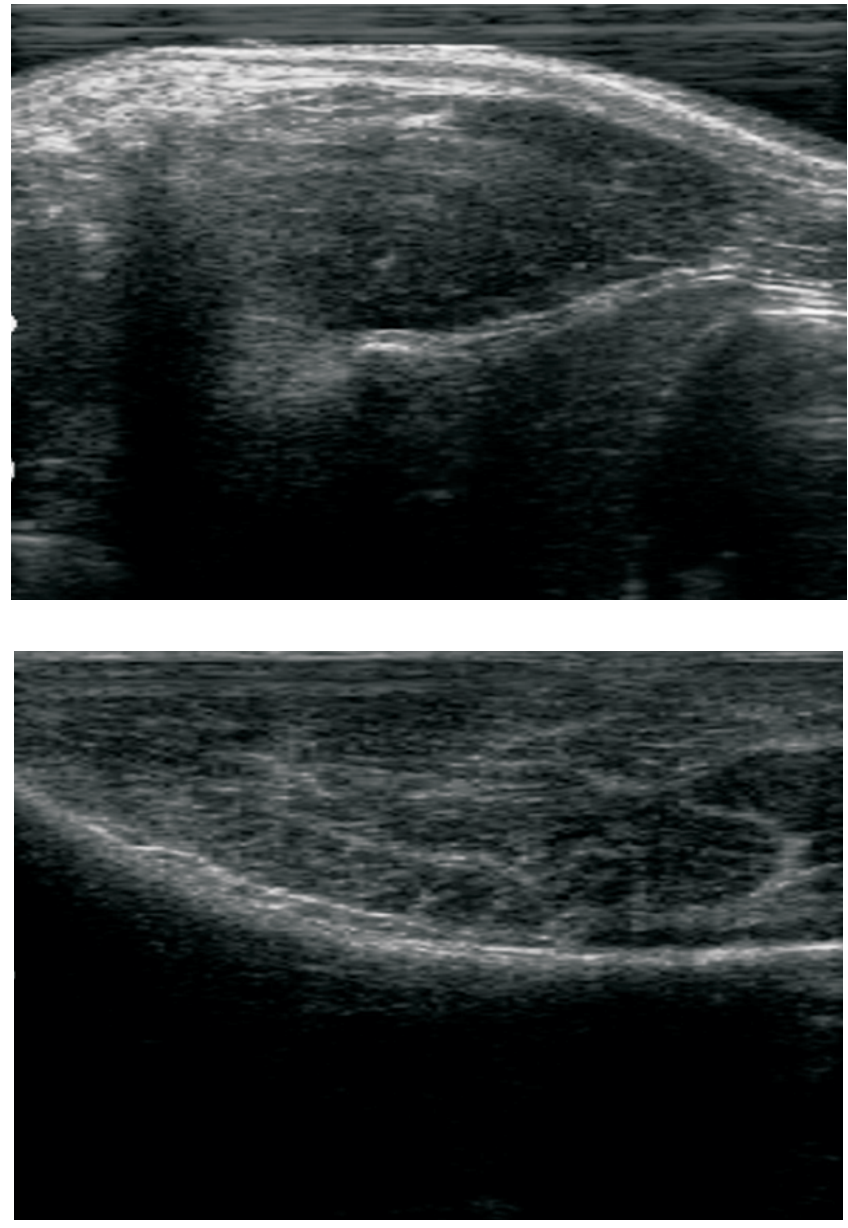

Figura 2 - Descrição dos pontos anatômicos de uma imagem obtida na região entre a $12^{\underline{a}}$ e a 13 a costelas (acima), classificada como marginal: difícil visualização do músculo spinalis dorsi e da borda medial do músculo longissimus dorsi; e de uma imagem obtida na região da picanha (abaixo), classificada como marginal: divisão entre os músculos biceps femoris e gluteus medius de difícil visualização.

$$
\sigma_{\mathrm{t}}^{2}=\frac{2[1+(\mathrm{n}-1) \mathrm{t}]^{2}(1-\mathrm{t})^{2}}{\mathrm{n}(\mathrm{n}-1)(\mathrm{N}-1)},
$$

em que: $\mathrm{n}$ = número de mensurações de cada imagem; e $\mathrm{N}=$ número de imagens envolvidas.

O uso da correlação como medida de repetibilidade é questionável, devido à sua dependência com a variância amostral (Hassen et al., 1998). Por essa razão, outra medida de repetibilidade utilizada foi a raiz do quadrado da soma dos erros, ou seja, o erro-padrão da diferença (SED) entre as duas mensurações:

$$
\mathrm{SED}=\sqrt{\frac{\sum\left(\mathrm{x}_{2}-\mathrm{x}_{1}\right)^{2}}{\mathrm{~N}}},
$$

em que $x_{1}$ e $x_{2}$ são a primeira e a segunda mensuração da mesma imagem e $\mathrm{N}$, o número de imagens. 
Para avaliar os efeitos de técnico e tipo de imagem, a diferença absoluta entre a primeira e a segunda mensuração de cada técnico para as três características (AOL, EGL e EGG) foi analisada pelo seguinte modelo:

$$
\mathrm{y}_{\mathrm{ijk}}=\mu+\mathrm{t}_{\mathrm{i}}+\mathrm{zj}+\mathrm{e}_{\mathrm{ijk}}
$$

em que: $y_{i j k}=$ ijk-ésima diferença absoluta entre a primeira e a segunda observação de cada técnico para AOL, EGL ou EGG; $\mu$ = média geral; $t_{i}=$ efeito fixo do i-ésimo técnico; $z_{j}=$ efeito fixo da j-ésima qualidade da imagem; $e_{i j k}=$ erro residual.

Para comparar a classificação das imagens de acordo com o técnico, foram computados coeficientes de correlação de Spearman entre a média das duas mensurações da imagem de cada técnico para cada uma das três características. Esse procedimento é importante para verificar se os dados para a seleção dos animais seriam os mesmos independentemente do técnico que mensurou a imagem no laboratório.

\section{Resultados e Discussão}

Considerando-se que o técnico 2 é o mais experiente, os técnicos 1 e 3 aparentemente subestimaram a AOL e superestimaram tanto a EGL como a EGG (Tabela 1). Entre a primeira e a segunda mensuração da imagem, houve pequenas diferenças entre as médias, contudo com tendência de maior amplitude para os dois técnicos com menor experiência ( 1 e 3 ).

Os valores médios observados para espessura de gordura subcutânea do lombo foram muito pequenos, devido à raça, à idade e, principalmente, ao sistema de produção dos animais. Animais jovens da raça Nelore criados em pastagem apresentam escassa cobertura de gordura, como descrito por Yokoo et al. (2008). A magnitude da característica influencia na precisão da medida e pode interferir na repetibilidade de mensurações dentro e entre técnicos (Robinson et al., 1992; Tarouco et al., 2005). Áreas de diferentes impedâncias acústicas entre tecidos (fáscias e tecido adiposo) provocam um artefato na imagem, que é o aumento da espessura da fáscia. Este aumento é devido à calibração do ganho total (amplificação da onda) em direção ao máximo, como também em tecidos que apresentem espessura menor que o comprimento de onda, o que ocorre com as fáscias e tecido adiposo com menos de $1 \mathrm{~mm}$ de espessura quando utilizadas frequências de 3,5 MHZ. Esses fatores dificultam a definição dos pontos a serem utilizados para a mensuração da espessura de gordura e podem influenciar nas diferenças entre técnicos.

A média da AOL está abaixo da relatada por Yokoo et al. (2008) para machos e fêmeas da raça Nelore de 15 a 20 meses de idade. As dificuldades encontradas para uma mensuração precisa e exata da AOL estão associadas à visualização dos pontos anatômicos importantes, como as laterais, que muitas vezes tem fraca definição e podem causar confundimento na delimitação do músculo, determinando aumento ou diminuição da AOL. A experiência do técnico e a padronização dos locais de posicionamento do cursor são importantes para a correta mensuração das imagens.

Sem exceção, as imagens classificadas como aceitáveis (com todos os pontos anatômicos de referência bem nítidos - Figura 1) foram as que apresentaram maior repetibilidade (Tabela 2), tanto entre como dentro de técnicos, com menores erros entre uma mensuração e outra (menores valores de SED), o que confirma a importância da habilidade do técnico em capturar uma boa imagem. Entretanto, mesmo com um conjunto de imagens aceitáveis a repetibilidade entre técnicos não foi tão alta (de 0,781 a 0,826). Perkins et al. (1992) observaram que a repetibilidade entre técnicos foi igual $(0,81)$ para AOL e EGL, mas Hassen et al. (1998) relataram repetibilidade entre técnicos inferior para $\mathrm{AOL}$ $(0,42)$ que para $\operatorname{EGL}(0,81)$.

Os valores de repetibilidade entre técnicos variaram de 0,826 a 0,588 , considerando ambas as classes de qualidade da imagem, e foram sempre superiores para a

Tabela 1 - Médias, por técnico, da área do músculo longissimus dorsi (AOL), espessura de gordura subcutânea no lombo (EGL) e na garupa (EGG) e das diferenças entre a $1 \underline{a}$ e a $2^{a}$ mensuração da imagem

\begin{tabular}{|c|c|c|c|c|c|c|}
\hline \multirow{3}{*}{$\begin{array}{l}\text { Item } \\
\text { Medidas }\end{array}$} & \multicolumn{6}{|c|}{ Técnico } \\
\hline & \multicolumn{2}{|c|}{1} & \multicolumn{2}{|c|}{2} & \multicolumn{2}{|c|}{3} \\
\hline & Média & Amplitude & Média & Amplitude & Média & Amplitude \\
\hline $\operatorname{AOL}\left(\mathrm{cm}^{2}\right)$ & $35,5 \pm 5,6$ & $23,0-55,3$ & $39,5 \pm 5,5$ & $26,7-56,6$ & $38,7 \pm 6,1$ & $25,5-59,5$ \\
\hline EGL (mm) & $0,8 \pm 0,8$ & $0-4,3$ & $0,7 \pm 1,0$ & $0-5,0$ & $0,9 \pm 0,6$ & $0-3,7$ \\
\hline EGG (mm) & $3,5 \pm 1,6$ & $0,8-7,9$ & $2,3 \pm 1,9$ & $0-7,0$ & $3,3 \pm 1,2$ & $1,2-6,1$ \\
\hline D-AOL $\left(\mathrm{cm}^{2}\right)^{1}$ & $1,6 \pm 1,4$ & $0-11,5$ & $1,1 \pm 0,9$ & $0-5,1$ & $2,2 \pm 1,9$ & $0-14,3$ \\
\hline D-EGL $(\mathrm{mm})^{1}$ & $0,2 \pm 0,3$ & $0-1,9$ & $0,2 \pm 0,3$ & $0-1,6$ & $0,3 \pm 0,2$ & $0-1,1$ \\
\hline D-EGG $(\mathrm{mm})^{1}$ & $0,3 \pm 0,3$ & $0-2,2$ & $0,3 \pm 0,5$ & $0-4,4$ & $0,1 \pm 0,2$ & $0-0,7$ \\
\hline
\end{tabular}

${ }^{1}$ Valor absoluto da diferença entre a primeira e a segunda mensuração da AOL, EGL e EGG. 
Tabela 2 - Medidas de repetibilidade, por classe de qualidade da imagem e técnico, das mensurações das imagens da área do músculo longissimus dorsi (AOL) e das espessuras de gordura subcutânea no lombo (EGL) e na garupa (EGG)

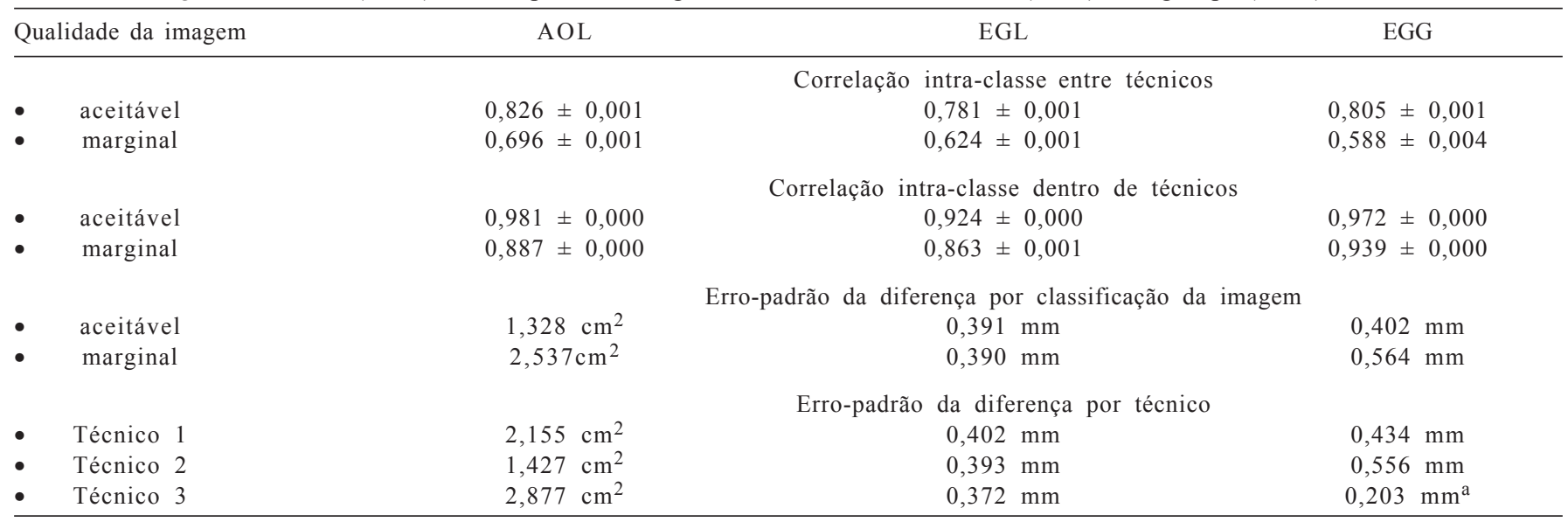

a Só imagens aceitáveis.

AOL. Hassen et al. (1998) alertam que a situação de repetibilidades não tão altas entre técnicos pode ter consequências adversas na avaliação de touros e que a inclusão do técnico no grupo de contemporâneos pode não ser suficiente para corrigir esse efeito.

As repetibilidades dentro de técnicos foram bem altas, principalmente para as imagens aceitáveis, o que confirma a importância de um só técnico interpretar as imagens coletadas em um grupo de contemporâneos. Considerando cada técnico separadamente, os valores de repetibilidade foram: 0,$898 ; 0,961$ e 0,$713 ; 0,882 ; 0,915$ e 0,534 ; e 0,938 e 0,915 para os técnicos 1,2 e 3 na mensuração de imagens de AOL, EGL e EGG classificadas como marginais (Figura 2), as quais apresentam alguma deficiência na definição dos pontos anatômicos de referência, mas podem ser interpretadas sem diminuir a precisão da medida. O técnico 3 não mensurou imagens de EGG de qualidade marginal. Para as imagens de AOL, EGL e EGG classificadas como aceitáveis, as repetibilidades foram 0,963;0,972 e 0,997; 0,$879 ; 0,943$ e 0,$961 ; 0,968 ; 0,967$ e 0,986 para os técnicos 1,2 e 3 .

Os erros dos técnicos entre uma e outra mensuração das imagens são expressos pelo erro-padrão da diferença (SED). O valor de SED mostrou tendência similar com os valores de repetibilidade para tipo de imagem, e os valores do técnico 2 (o mais treinado) foram os mais baixos para AOL e EGC, o que mostra a importância da experiência na mensuração das imagens. Hassen et al. (1998) relataram repetibilidades altas dentro de técnicos, sobretudo para $\operatorname{AOL}(0,96$ e 0,97$)$, com maiores valores para o técnico mais treinado. Os mesmos autores relataram maiores valores de erro-padrão da diferença para AOL (de 3,62 cm e 4,32 cm $\mathrm{cm}^{2}$ ) e menores para EGL $(0,09 \mathrm{~mm})$ em comparação aos deste trabalho (Tabela 2).
$\mathrm{Na}$ análise de variância das diferenças absolutas entre a primeira e a segunda mensuração da imagem, foram significativos $(p<0,05)$ os efeitos de técnico e tipo de imagem e a interação para AOL (coeficiente de determinação do modelo $\left.-\mathrm{R}^{2}=0,32\right)$ e somente de técnico para EGG $\left(\mathrm{R}^{2}=0,03\right)$. Para as diferenças absolutas da EGL, os efeitos de técnico e tipo de imagem não foram significativos $\left(\mathrm{R}^{2}=0,02\right)$.

As correlações entre a classificação das imagens dos animais entre técnicos (Tabela 3 ) foram todas significativas $(\mathrm{P}<0,01)$ e maiores para as medidas de AOL e EGG e mais baixas para EGL. Portanto, em uma avaliação genética, é possível que ocorra reclassificação dos animais, dependendo do técnico envolvido na mensuração da imagem, principalmente quanto à EGL .

Os resultados obtidos permitem afirmar que a repetibilidade das mensurações de imagens de características de carcaça obtidas por ultrassonografia certamente depende da qualidade da imagem, que, aliada à experiência do técnico,

Tabela 3 - Coeficientes de correlação de Spearman entre a média das duas mensurações da imagem

\begin{tabular}{|c|c|c|}
\hline \multirow[b]{2}{*}{ Item } & \multicolumn{2}{|c|}{ Técnico } \\
\hline & 2 & 3 \\
\hline & \multicolumn{2}{|c|}{$\mathrm{AOL}$} \\
\hline Técnico 1 & 0,95 & 0,81 \\
\hline \multirow[t]{2}{*}{ Técnico 2} & & 0,78 \\
\hline & \multicolumn{2}{|c|}{ EGL } \\
\hline Técnico 1 & 0,78 & 0,44 \\
\hline \multirow[t]{2}{*}{ Técnico 2} & & 0,50 \\
\hline & \multicolumn{2}{|c|}{ EGG } \\
\hline Técnico 1 & 0,92 & 0,91 \\
\hline Técnico 2 & & 0,79 \\
\hline
\end{tabular}

AOL: área do músculo longissimus dorsi; EGL: espessura de gordura subcutânea no lombo; EGG: espessura de gordura subcutânea na garupa. 
parece ter maior influência na repetibilidade da mensuração da AOL. As repetibilidades da mensuração de imagens ultrassônicas aceitáveis podem ser até 36\% maiores entre técnicos, e até $10 \%$ maiores dentro de técnicos, que a repetibilidade da mensuração de imagens de qualidade marginal.

\section{Conclusões}

A repetibilidade de mensurações de imagem de características de carcaça de bovinos de corte obtidas por ultrassonografia é maior dentro que entre técnicos, e depende da qualidade da imagem. Imagens classificadas como aceitáveis, com todos os pontos anatômicos facilmente identificáveis, possibilitam maior repetibilidade das medidas, comprovando a importância da qualidade da imagem no resultado final das avaliações. É recomendável que a mensuração de imagens de animais de mesmo grupo contemporâneo seja feita por um único técnico.

\section{Referências}

DIAS, F. Impactos do aumento de peso e acabamento da carcaça sobre os custos de processamento e valor comercial da carne de bovinos. In: SEMINÁRIO DE REVISÃO DOS CRITÉRIOS DE SELEÇÃO DAS RAÇAS ZEBUÍNAS, 2., Uberaba, 2006. Disponível em: <http://www.assocon.com.br/palestra_assocon2 $>$. Acesso em: 24/5/2007.

FALCONER, D.S.; MACKAY, T.F.C. Introduction to quantitative genetics. Essex: Longman, 1996. 464p.
HASSEN, A.; WILSON, D.E.; WILLHAM, R.L. et al. Evaluation of ultrasound measurements of fat thickness and longissimus muscle area in feedlot cattle: Assessment of accuracy and repeatability. Canadian Journal of Animal Science, v.78, p.277-285, 1998.

LÔBO, R.B.; BEZERRA, L.A.F.; OLIVEIRA, H.N. et al. Avaliação genética de touros e matrizes da raça Nelore: Sumário 2004. Ribeirão Preto, GEMAC - Departamento de Genética FMRP-USP, 2004. 122p.

PERKINS, T.L.; GREEN, R.D.; HAMLIN, K.E. et al. Ultrasonic prediction of carcass merit in beef cattle: Evaluation of technician effects on ultrasonic estimates of carcass fat thickness and longissimus muscle area. Journal of Animal Science, v.70, p.2758-2765, 1992.

ROBINSON, D.L.; McDONALD, C.A.; HAMMOND, K. et al. Live animal measures of carcass traits by ultrasound: assessment and accuracy of sonographers. Journal of Animal Science, v.70, p.1667-1676, 1992 .

TAROUCO, J.U.; LOBATO, J.F.P.; TAROUCO, A.K. et al. Relação entre medidas ultra-sônicas e espessura de gordura subcutânea ou área de olho de lombo na carcaça em bovinos de corte. Revista Brasileira de Zootecnia, v.34, n.6, p.2074-2084, 2005.

TAROUCO, J.U.; SOUZA, J.C.D.; ALMEIDA, J.C.C. Informações sobre a composição corporal "in vivo" em zebuínos obtidas pela técnica de ultra-sonografia "real-time". In: REUNIÃO ANUAL DA SOCIEDADE BRASILEIRA DE ZOOTECNIA, 30., 1993, Rio de Janeiro. Anais... Viçosa, MG: Sociedade Brasileira de Zootecnia, 1993. p.252.

WILSON, D.E. Centralized ultrasound processing. Study guides. Atlantic: Iowa State University, 1999. 167p.

WILSON, D.E. Ultrasound certification statistics. In: WORKSHOP DE ULTRA-SONOGRAFIA PARA AVALIAÇÃO DE CARCAÇA BOVINA, 2., 2006, Uberaba. Anais... Uberaba: AVAL Serviços Tecnológicos S/A, 2006. (CD-ROM).

YOKOO, M.J.I.; ALBUQUERQUE, L.G.; LÔBO, R.B. et al. Genetic and environmental factors affecting ultrasound measures of longissimus muscle area and backfat thickness in Nelore cattle. Livestock Science, v.116, p.147-154, 2008. 